\title{
Harsanyi Values of Large Economies: Nonequivalence to Competitive Equilibria*
}

\begin{abstract}
Sergiu Hart
Department of Economics, Department of Mathematics, and

Center for Rationality and Interactive Decision Theory, The Hebrew University of Jerusalem, Feldman Building, Givat-Ram, 91904 Jerusalem, Israel

and

Andreu Mas-Colell

Department of Economics, Harvard Univer sity, Cambridge, Massachusetts 02138, and Department of Economics, Univer sitat Pompeu Fabra, Barcelona, Spain

Received June 4, 1993

We consider economies with many agents and compare the competitive equilibria and the value allocations of the resulting coalitional games. In particular, we provide a (smooth and robust) example where the "value principle" does not hold for the Harsanyi NTU-value: there is a unique competitive equilibrium, which however does notbelong to the (nonempty) set of Harsanyi value allocations. Journal of Economic Literature Classification Numbers: C71, D51. ( 1996 Academic Press, Inc.
\end{abstract}

\section{INTRODUCTION}

Consider an economic situation of the standard general equilibrium variety. Such an economy determines the coalitional form of a cooperative game and one can then study the allocations that conform to some of the axiomatically based solution concepts of cooperative game theory. In this paper we focus on value concepts. The traditional justification of these is normative, but connections also exist with multilateral bargaining theory.

Suppose for a moment that utility is transferable (this is called the TU case). Then the central value solution is the Shapley (1953) value. Shapley and Shu-

* The authors thank the National Science Foundation and the U.S.-Israel Binational Science Foundation for financial support. We also thank an anonymous referee. 
bik (1969) and Aumann and Shapley (1974) established a remarkable result: provided that the economy has many traders and is smooth, the Shapley value allocation coincides with the unique competitive equilibrium. ${ }^{1}$ In parallel with the Core Equivalence Theorem, this is called the "Value Equivalence Theorem."

How general is the Value Equivalence Theorem, and, in particular, does it survive in a world without transferable utility (NTU)? Champsaur (1975), Aumann (1975), Mas-Colell (1977), and Hart (1977) (see Cheng, 1996, for a survey) established the following Value Principle: In a large economy all value allocations are competitive, and moreover, if the economy is sufficiently regular, then the two sets of allocations coincide. All these papers use an adaptation of the Shapley TU-value to the non-transferable utility case that was proposed by Shapley (1969) and is called the Shapley NTU-value. ${ }^{2}$

The purpose of this paper is to take a second look at this result by presenting an example that, at the very least, shows that the general validity of the Value Principle is under some question. In our (robust) example it does not hold. Specifically, in the example there is a unique Walrasian allocation, which does not however belong to the (non-empty) set of value allocations.

Since the results mentioned in the paragraph before last are of course correct, some explanation is required. The key to our approach is that we adopt a different generalization of the Shapley TU-value to the NTU context: the Harsanyi NTUvalue introduced by Harsanyi (1963). We do not claim that the Harsanyi value is the "ultimate" value, but we do believe that it is a more sophisticated adaptation to the NTU-case than the Shapley NTU-value. In a way which is illustrated by the current paper (for other ways see Roth, 1980; Shafer, 1980; and Hart, 1985), the Shapley procedure reduces the construction of the NTU-value to the TU-case in too drastic a manner.

We do not think that our position on the Harsanyi vs. the Shapley NTUvalues should be controversial. Shapley introduced his NTU-value explicitly as a simplification of the Harsanyi value. It then turned out to be of interest in its own right, and moreover amenable to analysis in economic models with many agents. In contrast, the construction of the Harsanyi value in large games is not a simple task. Yet by pursuing the potential approach introduced in Hart and Mas-Colell (1989), we have already carried out the necessary technical groundwork; see our investigation of the egalitarian solutions in Hart and Mas-Colell (1995a and 1995b) (to be referred to from now on as (HM,I) and (HM,II), respectively). Given this, constructing the Harsanyi values becomes a relatively easy task.

\footnotetext{
${ }^{1}$ More precisely: If "many traders" is modeled by a continuum, then there is a unique equilibrium (recall that we are in the TU-case), there is a unique value, and the two coincide. If one considers instead appropriate sequences of finite economies, then the limit of the values is precisely the unique competitive allocation.
}

${ }^{2}$ Known in the literature also as the " $\lambda$-transfer value." 
Finally, we note here that Imai (1983) has also offered an analysis of Harsanyi values of continuum games. We discuss his work in Section VII.

Sections II-IV are devoted to the definitional preliminaries and to an informal summary of the facts one needs to know from our earlier work. The example of a large economy whose unique Harsanyi NTU-value differs from its unique competitive allocation is presented in Section VI. Sections V and VII clarify the theoretical issues: they establish when and why the (near) equality of Harsanyi values and competitive equilibria can be expected. Section VIII is devoted to an informal discussion of some of the underlying economic considerations. Most of the technical details are relegated to three appendices.

\section{HARSANYI VALUES OF FINITE GAMES}

In this section we will recall the definition of the Harsanyi (1963) value of a finite game.

Let $(N, V)$ be a finite $N T U$-game in coalitional form. Here $N=\{1, \ldots, n\}$ is a finite set of players and $V$ is a point-to-set map that associates a set $V(S) \subset \mathbb{R}^{N}$ with every coalition $S \subset N$. The interpretation is that the elements of $V(S)$ are the payoff vectors that are achievableby the coalition $S$. We assume that the sets $V(S)$ are nonempty, closed, convex, and comprehensive. Also, $V(S)+\mathbb{R}^{N \backslash S} \subset V(S)$, i.e., the payoffs of players not in $S$ can take any value (this is just an inessential convention ${ }^{3}$ ).

The Harsanyi value is constructed in two steps. In the first, a vector of utility comparison weights $\lambda \in \mathbb{R}_{++}^{N}$ is taken as given. ${ }^{4}$ Thus, $\lambda_{i}$ may be interpreted as the "social" utility equivalent of a unit of utility of player $i$. Relative to these weights one considers then the "egalitarian" (to be called " $\lambda$-egalitarian") solution. This solution tries to capture the idea that the gains from cooperation are split equally among the players (hence comparison weights are needed); see Kalai and Samet (1985). We will present here a definition that fits particularly well with later developments. It is based on the potential approach developed in Hart and Mas-Colell (1989, Section 6).

Consider to begin with the case where all weights are equal, say $\lambda=(1,1$, $\ldots, 1)$. The potential function $P$ is the unique function $P: 2^{N} \rightarrow \mathbb{R}$ which satisfies $P(\emptyset)=0$ and

$$
D P(S):=(P(S)-P(S \backslash i))_{i \in S} \in \partial V(S) \quad \text { for all } S \subset N,
$$

where $\partial V(S)$ denotes the (Pareto efficient) boundary of $V(S)$, the coordinates of

\footnotetext{
${ }^{3}$ Note that here $V(S)$ is a subset of $\mathbb{R}^{N}$ and not a subset of $\mathbb{R}^{S}$. The two representations are equivalent (the coordinates of $N \backslash S$ are arbitrary), and the choice is just a matter of convenience.

${ }^{4}$ We write $\mathbb{R}_{++}^{N}$ for the strictly positive orthant of $\mathbb{R}^{N}$. The assumptions we make will ensure that, for the Harsanyi value, one need not consider the case where some of the weights vanish.
} 
$N \backslash S$ being ignored. The egalitarian solution of $(N, V)$ is then the payoff vector $E g(N):=D P(N) \in \partial V(N)$. To justify the term "egalitarian" note that for any $S \subset N$ and $i, j \in S$ we have

$$
E g_{i}(S)-E g_{i}(S \backslash j)=E g_{j}(S)-E g_{j}(S \backslash i) ;
$$

that is, relative to the solution, what $j$ contributes to $i$ is the same as what $i$ contributes to $j$ (the contribution of $j$ to $i$ being measured by the change in $i$ th payoff due to $j$ th presence). This "preservation of differences" principle could actually be taken as the defining axiom of the egalitarian solution (see Myerson, 1980, or Hart and Mas-Colell, 1989).

For a general $\lambda$, let $V_{\lambda}$ be the $\lambda$-rescaling of $V$, that is $V_{\lambda}(S):=\lambda * V(S)=$ $\{\lambda * a: a \in V(S)\}$ for all $S \subset N$, where we use the notation $\lambda * a:=\left(\lambda_{1} a_{1}, \lambda_{2} a_{2}\right.$, $\left.\ldots, \lambda_{n} a_{n}\right) \in \mathbb{R}^{N}$ for the coordinatewise multiplication of vectors. Then we define $a \in \partial V(N)$ to be the $\lambda$-egalitarian solution of $(N, V)$ if it is egalitarian relative to the units $\lambda$; i.e., if its rescaling $\lambda * a$ is the egalitarian solution of the rescaled game $\left(N, V_{\lambda}\right)$. Equivalently, define the $\lambda$-potential function $P_{\lambda}$ of $(N, V)$ as the potential function of the game $\left(N, V_{\lambda}\right)$ : it is the unique function $P_{\lambda}: 2^{N} \rightarrow \mathbb{R}$ which satisfies $P_{\lambda}(\emptyset)=0$ and

$$
D P_{\lambda}(S):=\left(P_{\lambda}(S)-P_{\lambda}(S \backslash i)\right)_{i \in S} \in \partial V_{\lambda}(S)=\lambda * \partial V(S) \quad \text { for all } S \subset N .
$$

The $\lambda$-egalitarian solution of $(N, V)$ is then that payoff vector $a \in \partial V(N)$ such that $\lambda * a=D P_{\lambda}(N)$ (i.e., $a=D P_{\lambda}(N) / \lambda$, where the division is again coordinatewise). We denote it by $E g_{\lambda} \equiv E g_{\lambda}(N)$.

The second step in the construction of the Harsanyi value consists in endogenizing the determination of the comparison weights $\lambda$. This is done by demanding that $\lambda$ be such that the $\lambda$-egalitarian solution $E g_{\lambda}$ be also $\lambda$-utilitarian, i.e., that it maximizes the sum of the $\lambda$-rescaled payoffs:

$$
\lambda \cdot E g_{\lambda} \geq \lambda \cdot a \quad \text { for all } a \in V(N) .
$$

If the boundary of $V(N)$ is smooth this can be expressed in local terms as the requirement that $\lambda$ reflect the marginal rates of transformation of utilities at the Pareto efficient point $E g_{\lambda}$. A Pareto efficient payoff vector $a \in \partial V(N)$ is a Harsanyi value if, when we compare utilities by means of some Pareto weights supporting $a$, it turns out that $a$ is egalitarian.

We summarize the definition: The payoff vector $a \in V(N)$ is a Harsanyi value of the game $(N, V)$ if there exists a vector $\lambda \in \mathbb{R}_{++}^{N}$ such that:

(i) $\lambda * a=D P_{\lambda}(N)$, where $P_{\lambda}$ is the $\lambda$-potential of $(N, V)$; and

(ii) $\lambda \cdot a \geq \lambda \cdot a^{\prime}$ for all $a^{\prime} \in V(N)$.

Note that the $\lambda$-eg alitarian solutions are not invariant to independent rescaling of utilities; Harsanyi values are. 
For weights chosen arbitrarily the $\lambda$-utilitarian solutions and the $\lambda$-egalitarian solutions will typically not coincide. To bring them into equality the weights $\lambda$ will have to be adjusted (informally speaking, the weights of players receiving comparatively less at the $\lambda$-utilitarian solution are to be increased, thus making their utility more valuable when looking at total utility and less necessary when trying to equalize social equivalents). Under our assumptions (see below), the $\lambda$-egalitarian solutions are individually rational (see, for example, Corollary V.2 in HM,II) and therefore belong to a compact subset of $\partial V(N)$. A standard fixed point argument then proves that the adjustment of weights can be done successfully and that therefore Harsanyi values exist.

In the transferable utility (TU) case (i.e., when there exists a real-valued function $v: 2^{N} \rightarrow \mathbb{R}$ such that, for all $S$, we have $a \in V(S)$ if and only if $\left.\sum_{i \in S} a^{i} \leq v(s)\right)$ there is a unique Harsanyi value: it is the Shapley (1953) value of the TU-game $(N, v)$ (the corresponding $\lambda$ is of course proportional to $(1,1, \ldots, 1))$. In the $n$-person pure bargaining case (i.e., when ${ }^{5} 0 \in \partial V(S)$ for all $S \neq N$ ) there is again a unique Harsanyi value; it is the Nash (1950) solution for the bargaining problem with feasible set $V(N)$ and disagreement point 0 . The Harsanyi value is thus an NTU solution that generalizes both of these classical concepts. There are other NTU values that do the same; in particular, see Shapley (1969) and Maschler and Owen (1992) (also Hart and Mas-Colell, 1996).

\section{HARSANYI VALUES OF CONTINUUM GAMES}

Our basic model of a game with a continuum of players is as follows. There are $n$ types of players. The primitive data are a set-valued map $V$ from $\mathbb{R}_{+}^{n}$ to the nonempty, closed, convex, and comprehensive subsets of $\mathbb{R}^{n}$. We call $V$ the game form (or, game function). Every $x \in \mathbb{R}_{+}^{n}$ (called a "profile") stands for a vector of the total masses of each of the $n$ types. Every $a \in V(x)$ is a vector of per-capita utility payoffs that are feasible for the profile $x$ (assuming equal treatment inside each type ${ }^{6}$ ). A continuum finite-type nontransferable utility game is given by specifying, in addition to $V$, the profile $\bar{x}$ of the grand coalition. Given the game form $V$, it is useful to introduce the notation $\hat{V}(x):=\{x * a: a \in V(x)\}$; vectors $b \in \hat{V}(x)$ represent total (per-type) payoffs.

In analogy with the case where there is only a finite number of players we again construct the Harsanyi value in two steps. In the first step a vector of (typesymmetric) utility comparison weights $\lambda \in \mathbb{R}_{++}^{n}$ is taken as given and, relative

\footnotetext{
${ }^{5}$ That is, the origin $0 \in \mathbb{R}^{N}$ is Pareto efficient for all coalitions that do not contain all players. Of course, we may take any fixed vector instead of 0 .

${ }^{6}$ We may restrict ourselves totype-symmetric allocations and weights since we are interested in the relations to the competitive allocations, which are type-symmetric. Allowing for non-type-symmetric values will not alter the results.
} 
to these weights, we consider the $\lambda$-eg alitarian solution. The task of defining the $\lambda$-egalitarian solutions for continuum games is not straightforward. We have provided the appropriate construction in our papers (HM,I and HM,II), together with extensive justifications. We emphasize again that there is nothing arbitrary in our definition: it is the one dictated by the asymptotics of finite game approximations. In Appendix A we list the precise set of hypotheses imposed in our two papers (HM,I and HM,II). These hypotheses are maintained throughout the present paper.

As shown in (HM,I), the construction of $\lambda$-egalitarian solutions requires the analysis of a first order partial differential equation. We summarize it now. For every $x \in \mathbb{R}_{++}^{n}$ and $p \in \mathbb{R}^{n}$, define

$$
v_{\lambda}(x, p):=\sup \{p \cdot(\lambda * a): a \in V(x)\},
$$

and also $\hat{v}_{\lambda}(x, p / x):=v_{\lambda}(x, p)$, where $p / x:=\left(p_{1} / x_{1}, \ldots, p_{n} / x_{n}\right) \in \mathbb{R}^{n}$. Thus $v_{\lambda}(x, \cdot)$ and $\hat{v}_{\lambda}(x, \cdot)$ are the support functions of the convex sets $V_{\lambda}(x):=$ $\lambda * V(x)$ and $\hat{V}_{\lambda}(x):=\lambda * \hat{V}(x)$, respectively. Note also that $v_{\lambda}(x, p)=v(x, \lambda * p)$. Consider for every $x \in \mathbb{R}_{++}^{n}$ the variational problem:

$$
P_{\lambda}(x):=\inf \int_{0}^{1} v_{\lambda}(\mathbf{x}(t), \dot{\mathbf{x}}(t)) d t,
$$

where the infimum is taken over all absolutely continuous paths $\mathbf{x}$ connecting the origin 0 to $x$, i.e., $\mathbf{x}:[0,1] \rightarrow \mathbb{R}_{++}^{n} \cup\{0\}$ with $\mathbf{x}(0)=0$ and $\mathbf{x}(1)=x$.

It turns out that the function $P_{\lambda}$ is well defined and Lipschitz (but not necessarily differentiable, even if the original data are as smooth as desired). Furthermore, whenever the gradient vector $\nabla P_{\lambda}(x)$ exists (which happens for almost every $x$ ) we have

$$
\nabla P_{\lambda}(x) \in \partial V_{\lambda}(x)
$$

We call the function $P_{\lambda}$ the (variational) $\lambda$-potential of $V$. In (HM,I) we also show that for smooth hyperplane games (i.e., games where $\hat{v}_{\lambda}(x, q)<\infty$ for a single normalized $q$, which may vary with $x$ ), and in particular for TU-games, the variational potential will be differentiable. As a matter of interpretation this means that the lack of differentiability of $P_{\lambda}$, which is a robust and non-pathological phenomenon, is essentially linked to the NTU character of the game. We note that the differentiability of $P_{\lambda}$ at $x$ is intimately related to the multiplicity of the optimal paths for the variational problem. In fact, $\nabla P_{\lambda}(x)$ exists if and only if the optimal path is unique (cf. Theorem $\mathrm{E}(\mathrm{c})$ in (HM,I)).

If the vector $a \in \partial V(x)$ satisfies $\lambda * a=\nabla P_{\lambda}(x)$ then it is clear that $a$ should be the $\lambda$-egalitarian solution for the game $(x, V)$. There is, however, a problem. What if $P_{\lambda}(x)$ is not differentiable at $x$ ? For fixed $\lambda$ this can only occur on a set of measure zero (since $P_{\lambda}$ is Lipschitz) and therefore in (HM,I), where 


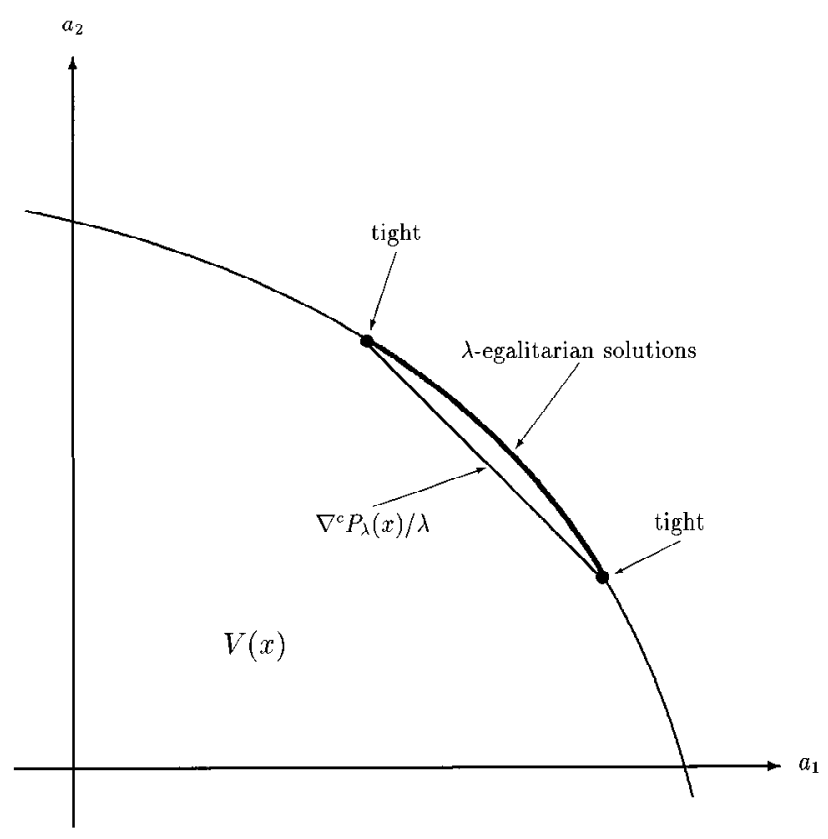

FIGURE 1

$\lambda$ was fixed, we did not worry about this issue. But in this paper we need to endogeneously adjust $\lambda$ and thus we cannot simply sidestep the problem. As we shall see below, those $\lambda$ 's such that $P_{\lambda}$ is not differentiable at the given $x$ may well be the crucial ones. What we will therefore do is to allow the egalitarian solutions to be set-valued at nondifferentiability points of the potential. We define an egalitarian solution as a Pareto efficient point that Pareto dominates some generalized gradient of the potential. Specifically, a payoff vector $a \in \partial V(x)$ is a $\lambda$-egalitarian solution for the game $(x, V)$ if $a \geq a_{0}$ for some $a_{0}$ such that $\lambda * a_{0} \in \nabla^{c} P_{\lambda}(x)$, where $\nabla^{c} P_{\lambda}(x)$ is the set of Clarke (1983) generalized gradients of $P_{\lambda}$ at $x$ (i.e., $\nabla^{c} P_{\lambda}(x)$ is the convex hull of the set $\left\{\lim \nabla P_{\lambda}\left(x_{m}\right): P_{\lambda}\right.$ is differentiable at $x_{m}$ and $x_{m} \rightarrow x$; ; see Fig. 1). Formally, the $\lambda$-egalitarian solution of the game $(x, V)$ is the set

$$
\operatorname{Eg}_{\lambda}(x, V):=\left(\nabla^{c} P_{\lambda}(x) / \lambda+\mathbb{R}_{+}^{n}\right) \cap \partial V(x) .
$$

When $P_{\lambda}$ is differentiable at $x, \operatorname{Eg}_{\lambda}(x, V)$ reduces to $\nabla P_{\lambda}(x)$ (see Corollary VII.4.3 in (HM,I)).

We moreover say that $a$ is a tight $\lambda$-egalitarian solution if actually $\lambda * a \in$ $\nabla^{C} P_{\lambda}(x)$. As we can see in Fig. 1 , if $\nabla^{c} P_{\lambda}(x)$ is not a singleton, then it may well contain nonefficient payoffs. The egalitarian solutions are always efficient. 


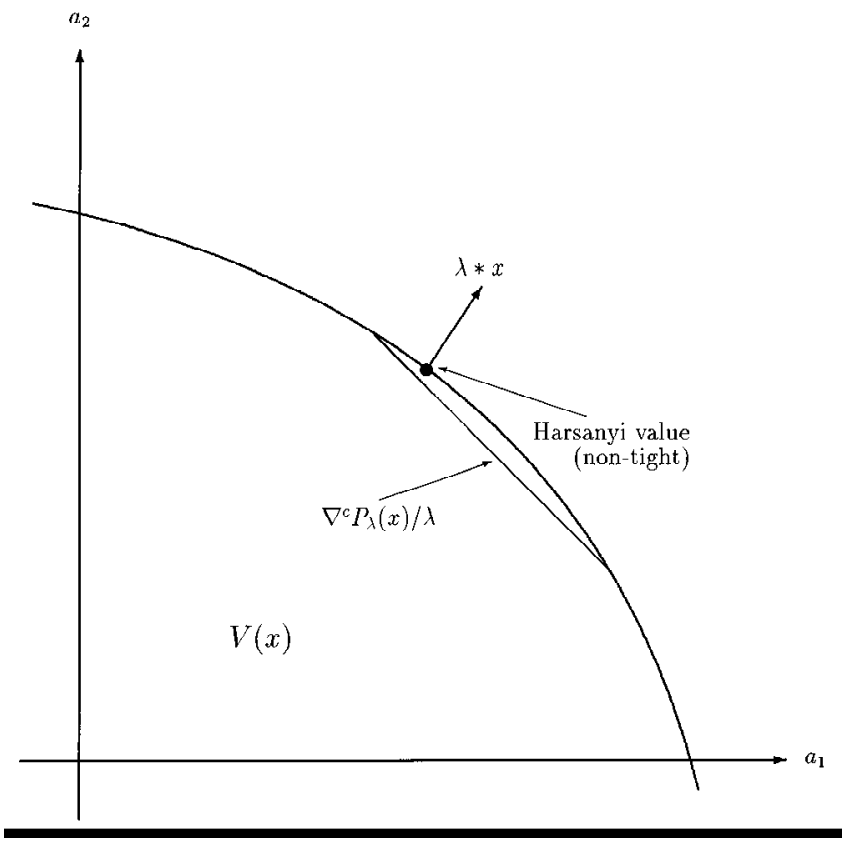

FIGURE 2

It is not difficult to see that for a TU-game the current construction (for equal weights across players) gives us the diagonal formula of Aumann and Shapley (1974) for the value of continuum TU-games. In fact, for any $x$ the solution to the variational problem is the diagonal path $\mathbf{x}(t)=t x$. Thus the gradient of the variational potential can be viewed as a natural extension of the diagonal formula to the NTU situation (see Sections VII and VIII below, and also Section IV.2 in (HM,I) for further discussions on this topic). We recall here a key feature: in the NTU case the optimal paths of the variational problem need not be diagonal.

The second step of the definition of the Harsanyi value consistsin endogenizing the determination of the weights. For this we proceed by imposing a $\lambda$-utilitarian requirement on the $\lambda$-egalitarian solution, in exactly the same manner as we did for the case with a finite number of players. We say that the payoff vector $a \in \partial V(x)$ is a Harsanyi value of the game $(x, V)$ if there is $\lambda \in \mathbb{R}_{++}^{n}$ such that:

(i) $a \geq a_{0}$ for some $a_{0}$ such that $\lambda * a_{0} \in \nabla^{c} P_{\lambda}(x)$; and

(ii) $\sum_{i} \lambda_{i} x_{i} a_{i} \geq \sum_{i} \lambda_{i} x_{i} a_{i}^{\prime}$ for all $a^{\prime} \in V(x)$.

If, in addition, $\lambda * a \in \nabla^{c} P_{\lambda}(x)$ then we say that $a$ is a tight Harsanyi value. See Fig. 2 for a nontight Harsanyi value. 
The previous definition, and in particular its use of the generalized gradient, may appear arbitrary. In the next section we put on record that it is the right definition, in the sense that it is given by the limits of the Harsanyi values of finite games approximations.

Another consequence of the result of the next section is a proof of the existence of Harsanyi values in the continuum case. A direct fixed-point proof (that is, not going through finite approximations) could also be given.

\section{HARSANYI VALUES OF LARGE BUT FINITE GAMES: CONVERGENCE}

Here we will show that the definition of the Harsanyi values in the previous section is the appropriate one, in the sense that it yields precisely the limits of the Harsanyi values of finite games converging to the limit continuum game. The definitions below follow (HM,II).

Given the continuum game $(\bar{x}, V)$, we obtain a sequence of finite games $\left(N_{r}, V_{r}\right)$, for $r=1,2, \ldots$, by regarding each player as having mass $1 / r$. More specifically, suppose that for every $r$ we are given a finite set of players $N_{r}$ which belong to some of the $n$ types. For a coalition $S \subset N_{r}$, let $m_{i}(S)$ be the number of players of type $i$ in $S$, and put $m(S):=\left(m_{1}(S), m_{2}(S), \ldots, m_{n}(S)\right) \in \mathbb{R}_{+}^{n}$. We will say that the finite game $\left(N_{r}, V_{r}\right)$ constitutes the $r$-approximation to the continuum game $(\bar{x}, V)$ if:

(i) the $r$-normalized profile $m\left(N_{r}\right) / r$ of the grand coalition is ${ }^{7} \bar{x}$; and

(ii) for each coalition $S \subset N_{r}$ the set of feasible type-symmetric payoff vectors, denoted by $V_{r}(S)$, is $V(m(S) / r)$.

Let $H_{r}$ denote the set of (type-symmetric) Harsanyi values of the finite game $\left(N_{r}, V_{r}\right)$; it is a subset of $\partial V_{r}\left(N_{r}\right)=\partial V(\bar{x})$. We can now state the convergence result.

THEOREM IV.1. Let $(\bar{x}, V)$ be a continuum game and let $\left(N_{r}, V_{r}\right)$ be its $r$ approximation. Then every limit point as $r \rightarrow \infty$ of the sets $H_{r}$ of Harsanyi values of the finite games $\left(N_{r}, V_{r}\right)$ is a Harsanyi value of the game $(\bar{x}, V)$.

The theorem will be proved in Appendix B; it is an application of the results and techniques developed in (HM,I) and (HM,II).

Our assumptions imply that each finite game $\left(N_{r}, V_{r}\right)$ possesses at least one Harsanyi value, and they all lie in the compact $\operatorname{set}^{8} \partial V(\bar{x}) \cap \mathbb{R}_{+}^{n}$. Therefore we

\footnotetext{
${ }^{7}$ We assume that $\bar{x}_{i}$ is an integer for each $i$ (for instance, $\bar{x}=(1, \ldots, 1)$ ).

${ }^{8}$ The $\lambda$-e galitarian solutions are individually rational—see, for example, Corollary V.2 in (HM,II) and therefore belong to $\partial V(\bar{x}) \cap \mathbb{R}_{+}^{n}$. A standard fixed-point argument then proves the existence of the Harsanyi values for the finite approximations. (Recall that both weights and payoffs are typesymmetric.)
} 
have as a corollary that Harsanyi values exist for the continuum game:

COROLlary IV.2. The continuum game $(\bar{x}, V)$ has a Harsanyi value.

\section{ECONOMIC GAMES WITH A CONTINUUM OF PLAYERS}

The remainder of the paper is devoted to the case where the game form $V$ is generated from an economy. The crucial property is homogeneity; namely, replicating the players does not change the feasible per capita payoffs. Standard models of pure exchange or production lead to such games.

We thus assume in addition to the standing hypotheses (again, see Appendix A) ${ }^{9}$

A.10 (Homogeneity). $\quad V(t x)=V(x)$ for all $x \in \mathbb{R}_{++}^{n}$ and $t>0$.

Equivalently, $\hat{V}(t x)=t \hat{V}(x)$ for all $x$ and $t$. Note that our standing hypotheses include superadditivity A.3, which, together with the homogeneity assumption A.10, yields concavity. That is, the supporting function $\hat{v}(x, q)$ is concave and homogeneous of degree 1 in $x$, for each fixed $q$. We will refer to such a $V$ as a market game form, and to $(\bar{x}, V)$ as a market game.

Let us note an immediate consequence of homogeneity:

LEMMA V.1. If the game form $V$ is homogeneous, then for each $\lambda \in \mathbb{R}_{++}^{n}$ the potential function $P_{\lambda}$ is homogeneous of degree 1.

Proof. Say that $\lambda=e=(1, \ldots, 1)$. For each $t>0$ define $Q^{t}(x):=P(t x) / t$ for all $x$. Then $Q^{t}$ is a Lipschitz potential (see (HM,I) for this concept). By the maximality property of the potential $P$ (see Theorem B(c) in (HM,I)) we get $P(x) \geq Q^{t}(x)=P(t x) / t$ for all $x$ and $t$. Substitute $x$ for $t x$ and $1 / t$ for $t$ to get the reverse inequality. Thus $P(x)=P(t x) / t$.

The economic solution concept we are interested in is that of the competitive or Walrasian equilibrium, which is based on the idea of allocating goods through prices that equalize demand and supply. We will not need, however, to introduce an explicit economic model. The reason is that we can appeal to the well known Core Equivalence Theorem, which asserts that in such economic games with a continuum of players the set of competitive equilibrium payoffs coincides with the core of the game (see Aumann, 1964). Thus, in investigating the relationship between the Harsanyi values and the competitive equilibria it suffices to compare the set of Harsanyi values to the core. Recall that a payoff vector $a \in \mathbb{R}^{n}$ is in the core of the continuum game $(\bar{x}, V)$ if $a \in \partial V(\bar{x})$ and $a \notin$ int $V(x)$ for all

\footnotetext{
${ }^{9}$ Note that A.10, like A.4-A.9, applies only to strictly positive profiles $x$. Also, from now on we assume A.3 rather than the weaker A.3w.
} 
$x \leq \bar{x}$; that is, $a$ is feasible and efficient for the grand coalition, and it can be improved upon by no subcoalition.

We are interested in the relations between the competitive equilibrium concept and the Harsanyi value. We will present first a "positive" result: tight Harsanyi values are competitive. Section VI will then provide an example where the unique Harsanyi value is nontight and it differs from the unique competitive allocation. In Section VII we analyze more deeply the relations between the two concepts.

THEOREM V.2. Let $(\bar{x}, V)$ be a continuum market game. Then every tight Harsanyi value belongs to the core, hence it is a competitive allocation of any underlying economy.

Proof. The result follows from our more detailed analysis of Section VII. We will provide here a simple (and hopefully instructive) proof for the case where the potential function is actually continuously differentiable everywhere.

Let $a \in \partial V(\bar{x})$ be a Harsanyi value of $(\bar{x}, V)$, with associated weights $\lambda \in$ $\mathbb{R}_{++}^{n}$. Thus $\lambda * a=\nabla P_{\lambda}(\bar{x})$, where $P_{\lambda}$ is the $\lambda$-potential.

The game is homogeneous, and so by Lemma V.1 the potential function $P_{\lambda}$ is homogeneous of degree 1 . Therefore $x \cdot \nabla P_{\lambda}(x)=P_{\lambda}(x)$ for all $x$ (by Euler's formula for homogeneous function). But $\nabla P_{\lambda} \in \lambda * V(x)$, which gives $x \cdot \nabla P_{\lambda}(x) \leq \hat{v}(x, \lambda)$, or $P_{\lambda}(x) \leq \hat{v}(x, \lambda)$ for all $x$. Moreover, at $\bar{x}$ we have equality, $P_{\lambda}(\bar{x})=\hat{v}(\bar{x}, \lambda)$, since $P_{\lambda}(\bar{x})=\bar{x} \cdot \nabla P_{\lambda}(\bar{x})=\bar{x} \cdot(\lambda * a)=$ $\lambda \cdot(\bar{x} * a)=\hat{v}(\bar{x}, \lambda)$, the last equality holding because $a$ is $\lambda$-utilitarian. Therefore $\nabla_{x} \hat{v}(\bar{x}, \lambda)=\nabla P_{\lambda}(\bar{x})=\lambda * a$ (two differentiable functions that coincide at $\bar{x}$ and such that one majorizes the other must have the same gradient at $\bar{x}$ ).

This yields $\hat{v}(x, \lambda) \leq \hat{v}(\bar{x}, \lambda)+\nabla_{x} \hat{v}(\bar{x}, \lambda) \cdot(x-\bar{x})=(\lambda * a) \cdot x$ for all $x$ (we used the concavity of $\hat{v}(\cdot, \lambda)$, and then applied Euler's formula at $\bar{x})$. Therefore $\lambda * a$ is weakly separated from $\lambda * V(x)$, or $a \notin \operatorname{int} V(x)$, for all $x$. Together with $a \in \partial V(\bar{x})$ this implies that $a$ belongs to the core of $(\bar{x}, V)$.

\section{THE EXAMPLE}

This section is devoted to the example of a market game whose (unique) Harsanyi value is not competitive.

There are two types (i.e., $n=2$ ). The coalitional function is

$$
\begin{aligned}
V(x):=\left\{a \in \mathbb{R}^{2}:\right. & 2 x_{1} a_{1}+x_{2} a_{2} \leq g\left(x_{1}, x_{2}\right), \\
& x_{1} a_{1}+2 x_{2} a_{2} \leq g\left(x_{1}, x_{2}\right) \\
& \left.3 x_{1} a_{1}+3 x_{2} a_{2} \leq 1.85 g\left(x_{1}, x_{2}\right)\right\},
\end{aligned} \text { and }
$$

where $g(x) \equiv g\left(x_{1}, x_{2}\right):=x_{1} x_{2} /\left(x_{1}+x_{2}\right)$. This can be rewritten in a more transparent form. Define

$$
B:=\left\{b \in \mathbb{R}^{2}: 2 b_{1}+b_{2} \leq 1, b_{1}+2 b_{2} \leq 1,3 b_{1}+3 b_{2} \leq 1.85\right\},
$$




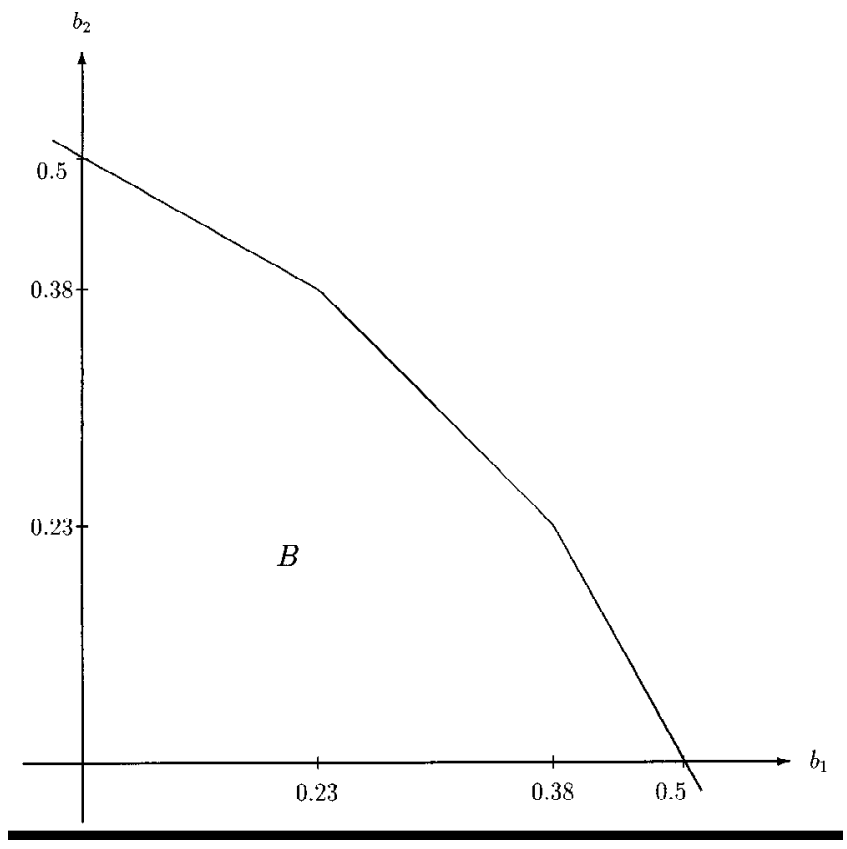

FIGURE 3

(see Fig. 3), then $\hat{V}(x)=g(x) B$ for all $x$. Note that $g$ is concave and homogeneous of degree 1 , and that the set $B$ is convex. Moreover, the support function $\hat{v}(x, \cdot)$ of $\hat{V}(x)$ satisfies $\hat{v}(x, \lambda)=g(x) w(\lambda)$ for all $x$ and $\lambda$, where $w$ is the support function of the set $B$.

This game form can be generated from an economy of the usual general equilibrium type. Suppose that there are two inputs, with amounts denoted $y_{1}$ and $y_{2}$, and two outputs, with amounts denoted $z_{1}$ and $z_{2}$. Initially each agent of type 1 owns just 1 unit of the first input (and nothing of the other goods); and each agent of type 2 owns just 1 unit of the second input. Thus the total initial endowment of a coalition with profile $\left(x_{1}, x_{2}\right)$ is $\left(x_{1}, x_{2}, 0,0\right)$. The utility functions of the two types depend only on outputs (and not on inputs). They are

$$
\begin{aligned}
& u_{1}\left(y_{1}, y_{2}, z_{1}, z_{2}\right):=\min \left\{z_{1} / 2, z_{2}\right\}, \\
& u_{2}\left(y_{1}, y_{2}, z_{1}, z_{2}\right):=\min \left\{z_{1}, z_{2} / 2\right\} .
\end{aligned}
$$

Finally, there is a constant returns to scale production technology, freely available to every coalition: the output vectors that are producible from inputs $\left(y_{1}, y_{2}\right)$ are

$$
\left\{\left(z_{1}, z_{2}\right): z_{1} \leq g\left(y_{1}, y_{2}\right), z_{2} \leq g\left(y_{1}, y_{2}\right), z_{1}+z_{2} \leq 1.85 g\left(y_{1}, y_{2}\right)\right\} .
$$


It is straightforward to check that this economy does indeed generate the game form $V$. If so desired it is possible to transform this economy into a pure exchange market that generates the same ${ }^{10} V$. The production interpretation, however, seems simpler and more natural.

We take $\bar{x}=(51,49)$ as the profile of the grand coalition. Any $\bar{x}$ sufficiently close to the diagonal but not on it will do (when $\bar{x}_{1}=\bar{x}_{2}$ the symmetry of the example makes all solutions identical).

Consider first the core. We claim that it consists of the single payoff vector

$$
\bar{c}:=\frac{1.85}{3} \nabla g(\bar{x}) \approx(0.148,0.160) .
$$

In Fig. 4 we place $\bar{x} * \bar{c}$ in $\hat{V}(\bar{x})$. It does indeed belong to $\partial \hat{V}(\bar{x})$ and the corresponding supporting Pareto weights are $\bar{\lambda}=(1,1)$. To verify that $\bar{c}$ belongs to the core recall that $\hat{v}(x, \lambda)=g(x) w(\lambda)$ for all $x$ and $\lambda$ (with $w$ the support function of the set $B$ ), and so $\nabla_{x} \hat{v}(\bar{x}, \bar{\lambda})=\nabla g(\bar{x}) w(\bar{\lambda})=\bar{c}$, because $w(\bar{\lambda})=1.85 / 3$. Since $\hat{v}(\cdot, \lambda)$ is concave and homogeneous of degree 1 we have

$$
\hat{v}(x, \bar{\lambda}) \leq \hat{v}(\bar{x}, \bar{\lambda})+\nabla_{x} \hat{v}(\bar{x}, \bar{\lambda}) \cdot(x-\bar{x})=\bar{c} \cdot x
$$

for all $x$, and therefore $\bar{c} \notin$ int $V(x)$ for all $x$. Thus $\bar{c}$ indeed belongs to the core. For uniqueness we refer to part (C2) of Theorem VII.1 below. ${ }^{11}$

Next we show that $\bar{c}$ is not a Harsanyi value. If it were, then the utilitarian condition in the definition of the Harsanyi value would imply that the vector of weights $\lambda$ must be (proportional to) $\bar{\lambda}=(1,1)$. For these weights we can exhibit

${ }^{10}$ This can be done by means of the so-called Rader's trick. Let the endowments be the same as above and the utility of each consumer be defined over net trades of the four commodities. Allow also negative trades of the output commodities (this is an inessential feature; it can be fixed by giving consumers positive endowments of output commodities). Make then the utility value of a net trade the maximum utility that the consumer can reach by using the available technology (thus transformed into a household technology). To verify that the game function is the same, note that what effectively goes on in computing $V(x)$ is that, say, type 1 consumers pass their inputs to type 2 consumers, who then produce by themselves and transfer output to the type 1 consumers.

${ }^{11}$ An economic argument for uniqueness goes as follows. Consider the production economy described in the text as underlying the gameform. We argue that it has a unique equilibrium. To this effect note, first, that at any Walrasian equilibrium the input prices have to be collinear with the marginal productivity vector $\nabla g(\bar{x})$. Therefore the distribution of income across types has to be same at all competitive equilibria. But it is well known that if the technology is of constant returns to scale and the consumers' preferences are homothetic then no two different equilibria are compatible with the same distribution of income. Hence the competitive equilibrium is unique, and therefore the core of the game consists of a single payoff vector. 


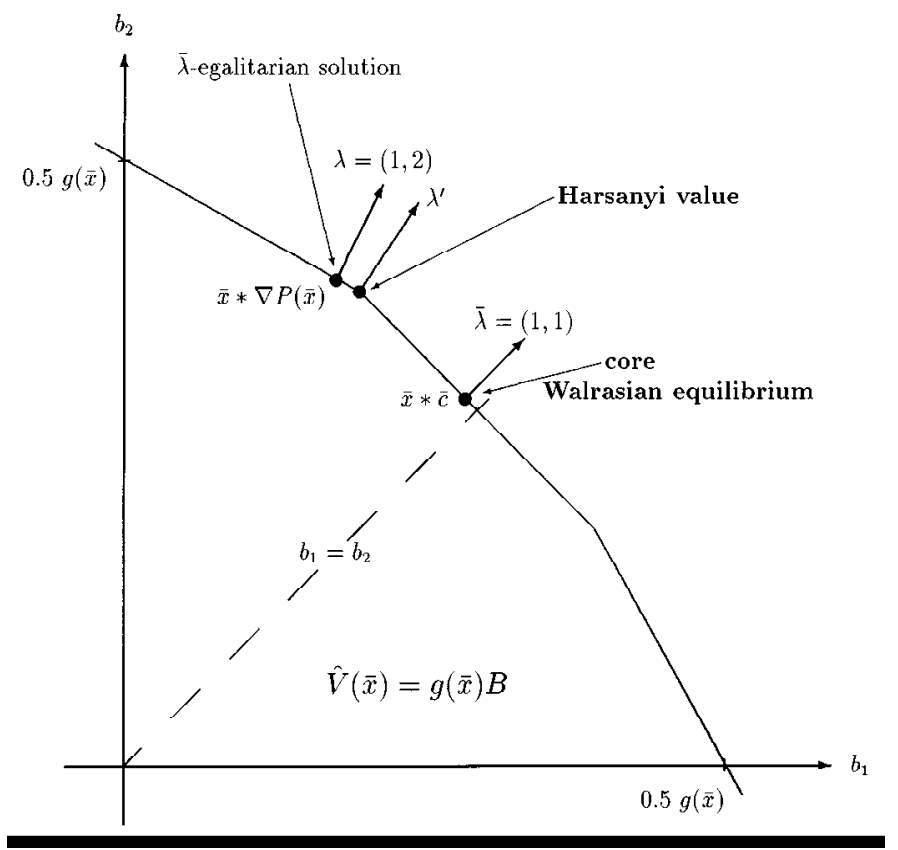

FIGURE 4

the potential explicitly (we write $P(x)$ for $P_{\bar{\lambda}}(x)$ ):

$$
P(x)= \begin{cases}x_{1}-\frac{\left(x_{1}\right)^{2}}{x_{2}} \ln \left(1+\frac{x_{2}}{x_{1}}\right), & \text { if } x_{1} \geq x_{2}, \\ x_{2}-\frac{\left(x_{2}\right)^{2}}{x_{1}} \ln \left(1+\frac{x_{1}}{x_{2}}\right), & \text { if } x_{1} \leq x_{2} .\end{cases}
$$

The function $P$ is differentiable everywhere except on the diagonal $x_{1}=x_{2}$. To verify that $P$ is the potential function one needs to check that for any point not on the diagonal, i.e., for any $x$ with $x_{1} \neq x_{2}$, one has:

(i) $\nabla P(x) \in \partial V(x)$; and

(ii) $P(x)=\int_{0}^{1} v(\mathbf{x}(t), \dot{\mathbf{x}}(t)) d t$ for some path $\mathbf{x}(\cdot)$ ending at $x$;

(see implication (ii) of Proposition V.7.1 in (HM,I)). Property (i), which is all it would take if $P$ were everywhere differentiable, can be checked by direct computation. Property (ii) is also not difficult to verify. We refer the reader to Section VIII of (HM,I) where these steps are carefully carried out for essentially the same example (more on this below). 
The $\bar{\lambda}$-egalitarian solution at $\bar{x}$ is therefore

$$
\nabla P(\bar{x}) \approx(0.108,0.199) \neq \bar{c} .
$$

Hence $\bar{c}$, the unique core allocation, cannot be a Harsanyi value. Thus we conclude that for this example no Harsanyi value (whose existence is guaranteed by Corollary IV.2) belongs to the core. In particular, by Theorem IV.1, no limit point of the set of Harsanyi values of the finite games $\left(N_{r}, V_{r}\right)$ belongs to the core.

This completes the example. It may be useful now to discuss the idea behind it. The starting point is the potential function $P$. We introduced this function in Section VIII of (HM,I) as an example of a nondifferentiable potential (for $\lambda=(1,1))$. The game function used there was $\hat{V}^{\prime}(x)=g(x) B^{\prime}$, with

$$
B^{\prime}=\left\{b \in \mathbb{R}^{2}: 2 b_{1}+b_{2} \leq 1, b_{1}+2 b_{2} \leq 1\right\} .
$$

(Note that the only difference is that the set $B$ is replaced by the slightly larger set $B^{\prime}$.) Whenever $P$ is differentiable at $x$ we have $x * \nabla P(x) \in \partial \hat{V}^{\prime}(x)$ but, precisely because of the nondifferentiability of $P$ along the diagonal $x_{1}=x_{2}$, it follows that $x * \nabla P(x)$ is bounded aw ay from the corner of $\partial \hat{V}^{\prime}(x)$, that is, from the point $g(x)(1 / 3,1 / 3)$. Hence we can "flatten" slightly the sets $\hat{V}^{\prime}(x)$ around this central corner while having $P$ remain the potential. This is what the set $B$ above does.

Suppose for a moment that we start at $x=(50,50)$. By symmetry the unique core payoff vector $c=\left(c_{1}, c_{2}\right)$ gives the same utility to the two types. Therefore $x * c$ is placed at the center of the middle segment of $\partial \hat{V}(x)$. Again by symmetry this $c$ is also the Harsanyi value. But it has to be a nontight Harsanyi value since the utilitarian weights are $\lambda=(1,1)$ and we know that if $\nabla P(x)$ existed then $x * \nabla P(x)$ could not belong to the middle segment. Let us now alter $x$ slightly to an $\bar{x}$ with unequal weights; $\bar{x}=(51,49)$ suffices. By continuity, for the unique new core payoffs $\bar{c}$, the vector $\bar{x} * \bar{c}$ will remain in the middle segment of $\partial \hat{V}(\bar{x})$ and therefore the only compatible utilitarian weights continue to be $\lambda=(1,1)$. But now $\bar{x}_{1} \neq \bar{x}_{2}$ implies that $P$ is differentiable at $\bar{x}$ and so $\bar{x} * \nabla P(\bar{x})$ does not lie in the middle segment. Our conclusion is that $\bar{c}$ cannot be a Harsanyi allocation.

Where are the Harsanyi values located? Note from Fig. 4 that $\bar{x} * \nabla P(\bar{x})$ lies in the part of the boundary of $V(\bar{x})$ with support vector $\lambda=(1,2)$ and that therefore the $\bar{\lambda}$ - utilitarian solutions give less to type 2 than the $\bar{\lambda}$-egalitarian solution (recall that $\bar{\lambda}=(1,1))$. In order to find a Harsanyi value this suggests increasing the weight of type 2 (thus making the utility of type 2 more valuable when we look at total social utility, and less necessary when we try to equalize social utility values across types). Indeed, it can be checked that there is a unique Harsanyi value (hence a unique limit of the Harsanyi values of the finite games $\left(N_{r}, V_{r}\right)$ ) at the "corner" point $\approx \bar{x} *(0.117,0.192)$, corresponding to some $\lambda^{\prime}$ between 


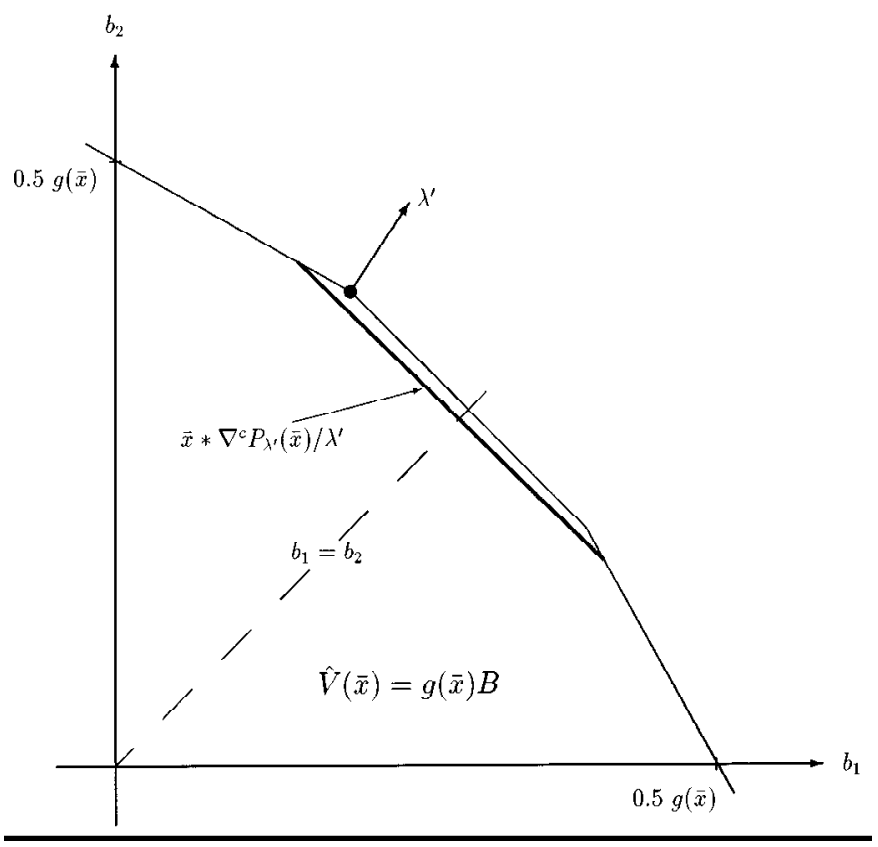

FIGURE 5

$(1,2)$ and $(1,1)$. At this $\lambda^{\prime}$ the potential $P_{\lambda^{\prime}}$ is not differentiable at $\bar{x}$ (when we change $\lambda$ from $(1,1)$ to $\lambda^{\prime}$ the potential function and its nondifferentiability region also change) and, as we already know from Theorem V.2, it must be the case that the Harsanyi value fails to be tight. See Fig. 5.

We conclude by observing that the example does not satisfy allour assumptions A.1-A.10 (see Appendix A). In particular, the boundaries of the sets $V(x)$ have both "flats" and "kinks." However, as the discussion above suggests, the example has been constructed to be robust. A close enough approximation making the boundaries both smooth and strictly convex will not matter: the core and the Harsanyi value still obtain at distinct points. For a specific such approximation, see Appendix C.

\section{NTU VALUES AND THE CORE}

In this section we analyze in more detail the connections between the core and the Harsanyi value.

An important element of the characterizations we will obtain is the diagonal path for $\bar{x}$, namely the path $\mathbf{x}:[0,1] \rightarrow \mathbb{R}_{+}^{n}$ defined by $\mathbf{x}(t):=t \bar{x}$ for all $t$. Given 
a vector $\lambda \in \mathbb{R}_{++}^{n}$, consider the variational problem that defines the $\lambda$-potential $P_{\lambda}$. We will say that the diagonal path for $\bar{x}$ is $\lambda$-minimal if $P_{\lambda}(\bar{x})$ is attained at this path (i.e., it solves the variational problem). We will say that the diagonal path for $\bar{x}$ is $\lambda$-critical if the Euler-Lagrange first order necessary conditions for the variational problem (cf. Theorem E(a) in (HM,I)) are satisfied along the diagonal path; thus, if the diagonal path is $\lambda$-minimal then it is $\lambda$-critical (but the converse need not hold, as we shall see below; the variational problem is not "convex").

Given an efficient payoff vector $a \in \partial V(\bar{x})$, we will say that the vector of normalized weights ${ }^{12} \lambda \in \Delta(N)$ is associated to $a$ if $a$ is $\lambda$-utilitarian. This may be formally expressed as $\lambda \cdot(\bar{x} * a) \equiv \bar{x} \cdot(\lambda * a)=\hat{v}(\bar{x}, \lambda)$ or equivalently, as $a=\nabla_{p} v(\bar{x}, \lambda * \bar{x})$. Note that our assumptions on the boundary of $V(\bar{x})$ imply that there is a one-to-one correspondence between $a$ and $\lambda$. We can now characterize the core.

THEOREM VII.1. Suppose that $(\bar{x}, V)$ is a continuum market game. Let a $\epsilon$ $\partial V(\bar{x})$ and let $\lambda \in \Delta(N)$ be the associated vector of normalized weights. Then the following three statements are equivalent:

(C1) a belongs to the core of $(\bar{x}, V)$;

(C2) $\lambda * a=\nabla_{x} \hat{v}(\bar{x}, \lambda)$

(C3) the diagonal path for $\bar{x}$ is $\lambda$-critical.

Proof. The Euler-Lagrange equation for the diagonal path is

$$
\nabla_{x} v(t \bar{x}, \lambda * \bar{x})=\frac{d}{d t}\left(\nabla_{p} v(t \bar{x}, \lambda * \bar{x})\right)
$$

for all $t \in(0,1]$. The right-hand side is the derivative with respect to $t$ of a constant (by homogeneity), hence it equals 0 . The left-hand side at $t=1$ equals $\nabla_{x} \hat{v}(\bar{x}, \lambda)-\lambda * \nabla_{p} v(\bar{x}, \lambda * \bar{x})=\nabla_{x} \hat{v}(\bar{x}, \lambda)-\lambda * a$ (we have used the identity $\hat{v}(x, q)=v(x, q * x)$; compare with the proof of Proposition VII.3.1 in(HM,I)). This establishes the equivalence of (C2) and (C3).

Next, assume (C2). Since $\hat{v}(\cdot, \lambda)$ is concave and homogeneous of degree 1 we have $\hat{v}(x, \lambda) \leq \hat{v}(\bar{x}, \lambda)+\nabla_{x} \hat{v}(\bar{x}, \lambda) \cdot(x-\bar{x})=(\lambda * a) \cdot x$ for all $x$. Therefore $\lambda * a$ is weakly separated from $\lambda * V(x)$, or $a \notin$ int $V(x)$. Thus $a$ belongs to the core.

Conversely, assume (C1): $a$ belongs to the core. Recall the "Hamiltonian" function $H$ introduced in Section VII.2 of (HM,I): $H\left(x, a^{\prime}\right):=\min \{v(x, p)-$ $\left.p \cdot a^{\prime}\right\}$, where the minimum is over all $p$ with $\|p\|=\|x\|$. We always have $\nabla_{x} H\left(x, a^{\prime}\right)=\nabla_{x} v(x, p)$ where $p$ is the solution of the minimum problem at $\left(x, a^{\prime}\right)$. Also, $H\left(x, a^{\prime}\right) \geq 0$ if and only if $a^{\prime} \in V(x)$, and $H\left(x, a^{\prime}\right)=0$ if and only if $a^{\prime} \in \partial V(x)$. Since $a \in$ core $(\bar{x}, V)$ we have $H(\bar{x}, a)=0$ and $H(x, a) \leq 0$

${ }^{12} \Delta(N)$ denotes the $n-1$ dimensional unit simplex on $N$, i.e., $\Delta(N)=\left\{\lambda \in \mathbb{R}_{+}^{N}: \sum_{i \in N} \lambda_{i}=1\right\}$. 
for all $x \leq \bar{x}$, hence for all $x$ (use homogeneity; for every $x$ there is $\rho>0$ such $\rho x \leq \bar{x})$. Therefore $H(\cdot, a)$ is maximal at $\bar{x}$, which implies that $\nabla_{x} H(\bar{x}, a)=0$. Let $\bar{p}=\lambda * \bar{x}$. Then $v(\bar{x}, \bar{p})=a \cdot \bar{p}$, and we have

$$
\nabla_{x} \hat{v}(\bar{x}, \lambda)=\nabla_{x} v(\bar{x}, \bar{p})+\lambda * \nabla_{p} v(\bar{x}, \bar{p})=\nabla_{x} H(\bar{x}, a)+\lambda * a=\lambda * a,
$$

or $(\mathrm{C} 2)$.

The equivalence $(\mathrm{C} 1) \Leftrightarrow(\mathrm{C} 2)$ should come as no surprise. It is the "Value Equivalence Theorem" for the Shapley NTU-value (see Shapley and Shubik, 1969, and Aumann, 1975). The Shapley NTU-value is defined as follows: A payoff vector $a \in \partial V(\bar{x})$ is a Shapley $N T U$-value of the game $(\bar{x}, V)$ if there exists a vector $\lambda \in \mathbb{R}_{++}^{n}$ of weights such that $\lambda * a$ equals the Aumann-Shapley (1974) TU-value of the continuum TU-game with coalitional function $\hat{v}(\cdot, \lambda)$ and grand coalition $\bar{x}$. We have

COROLlary VII.2. Let $(\bar{x}, V)$ be a continuum market game. Then the core coincides with the set of Shapley NTU-values.

Proof. The Aumann-Shapley TU-value of the TU-game $(\bar{x}, \hat{v}(\cdot, \lambda))$ is $\int_{0}^{1} \nabla_{x} \hat{v}(t \bar{x}, \lambda) d t$ (by the "diagonal formula"), which equals $\nabla_{x} \hat{v}(\bar{x}, \lambda)$ since $\hat{v}(\cdot, \lambda)$ is homogeneous of degree 1. Therefore $a$ is a Shapley NTU-value if and only if (C2) is satisfied.

We may thus add to Theorem VII.2 another statement that is equivalent to (C1), (C2), and (C3), namely:

(C4) a is a Shapley NTU-value of $(\bar{x}, V)$.

We come now to the Harsanyi value.

THEOREM VII.3. Suppose that $(\bar{x}, V)$ is a continuum market game. Let a $\in$ $\partial V(\bar{x})$ and let $\lambda \in \Delta(N)$ be the associated vector of normalized weights. Then the following three statements are equivalent:

(H1) $a$ is a tight Harsanyi value of $(\bar{x}, V)$;

(H2) $P_{\lambda}(\bar{x})=\hat{v}(\bar{x}, \lambda)$;

(H3) the diagonal path for $\bar{x}$ is $\lambda$-minimal.

Proof. For the diagonal path we have $v(\mathbf{x}(t), \lambda * \dot{\mathbf{x}}(t))=v(t \bar{x}, \lambda * \bar{x})=$ $v(\bar{x}, \lambda * \bar{x})=\hat{v}(\bar{x}, \lambda)$ for all $t$ (we used homogeneity). This yields the equivalence of $(\mathrm{H} 2)$ and $(\mathrm{H} 3)$.

Next, assume (H1): $a$ is a tight Harsanyi value. The potential function $P_{\lambda}$ is homogeneous of degree 1 . Therefore at every $x$ where it is differentiable we have $P_{\lambda}(x)=\left(P_{\lambda}(x+\delta x)-P_{\lambda}(x)\right) / \delta \rightarrow x \cdot \nabla P_{\lambda}(x)$ as $\delta \rightarrow 0^{+}$. Thus $P_{\lambda}(x)=x \cdot \nabla P_{\lambda}(x)$ at all differentiability points of $P_{\lambda}$, implying that $P_{\lambda}(x)=$ 
$x \cdot a^{\prime}$ for every $x$ and every (Clarke) generalized gradient $a^{\prime} \in \nabla^{c} P_{\lambda}(x)$. In particular, since $\lambda * a \in \nabla^{c} P_{\lambda}(\bar{x})$ (here we use the tightness assumption), we have $P_{\lambda}(\bar{x})=\bar{x} \cdot(\lambda * a)$, which equals $\hat{v}(\bar{x}, \lambda)$ since $a$ is $\lambda$-utilitarian. This is (H2).

For the converse, assume (H2). For every $x$ we have $v(\mathbf{x}(t), \lambda * \dot{\mathbf{x}}(t))=$ $v(t x, \lambda * x)=v(x, \lambda * x)=\hat{v}(x, \lambda)$ for the diagonal path for $x$, implying that $P_{\lambda}(x) \leq \hat{v}(x, \lambda)$. At $\bar{x}$ there is equality (this is (H2)), and so $P_{\lambda}(x)-\hat{v}(x, \lambda)$ is maximized at $x=\bar{x}$. Hence 0 is a generalized gradient of this function at $\bar{x}$ (cf. Proposition 2.3.2 of Clarke, 1983), or $\nabla_{x} \hat{v}(\bar{x}, \lambda) \in \nabla^{c} P_{\lambda}(\bar{x})$. But (H2) implies (C2) (since $\lambda$-minimal (H3) implies $\lambda$-critical (C3); see Theorem E(a) of (HM,I)). Therefore $\lambda * a=\nabla_{x} \hat{v}(\bar{x}, \lambda) \in \nabla^{c} P_{\lambda}(\bar{x})$ and $a$ is a tight Harsanyi value.

Note finally that from Theorems VII.1 and VII.2 we immediately obtain (since (H3) implies (C3)) Theorem VI.2: Every tight Harsanyi value is in the core.

This is an appropriate time to compare our results with those of Imai (1983). For continuum games similar to the ones considered in this paper, Imai defines a notion of "diagonal $H$-value" and proves its equivalence, in the homogeneous case, to the core. In our terms, Imai's value corresponds to a payoff vector $a \in \partial V(\bar{x})$ with an associated vector of weights $\lambda$ having the property that the diagonal path from $\bar{x}$ is $\lambda$-critical. Thus, Imai's equivalence result is in the nature of our Theorem VII.1. It is therefore an implication of our example that the limit of the finite games' Harsanyi values need not be an Imai diagonal $H$-value. That is, at the limit $\lambda$, the diagonal path may not be $\lambda$-critical. There always exists a $\lambda$-critical path-the minimal path for the $\lambda$-variational problem-but, as in our example, this path may be not diagonal.

We emphasize that these differences are not a matter of taste in definitions. The only notion of Harsanyi value for the continuum case that precisely captures the limits of the finite games approximations is the one we have given in this paper. And, as we have seen, it need not coincide with the solution suggested by Imai.

\section{DISCUSSION}

The content of Section VII constitutes the beginning of an understanding of the failure of the Value Equivalence Theorem for the Harsanyi value from the standpoint of its formal, mathematical structure. But from the economic point of view there is still much to understand. We devote this section to a few imprecise remarks.

The fact that theequivalence theorem holds for TU-g ames shows that nonequivalence is intimately related to the lack of perfect substitutability among players' utilities. It can be presumed that the failure may be more likely the farther we are from perfect substitutability. 
If we look at the example of Section VI from the economic standpoint we detect at least two sources of nonsubstitutability:

(i) There is no perfect substitutability of inputs (or, to be more precise, there is no perfect substitutability of the input contributions of the two types). The production function of the example can be written as $g(x)=\left(x_{1}^{-1}+x_{2}^{-1}\right)^{-1}$. It is a CES withelasticity of substitution $1 / 2$, thus less than 1 . Suppose we were to try a Cobb-Douglas production function, $g(x)=\sqrt{x_{1} x_{2}}$. Then $P(x)=(1.85 / 3) g(x)$ is the potential (for $\bar{\lambda}=(1,1)$ ) since $x * \nabla P(x)=(1.85 / 3) g(x)(1 / 2,1 / 2) \in$ $g(x) \partial B$ for all $x$. Since the function $P$ is differentiable we have that competitive equilibria and Harsanyi values coincide. It may be conjectured that this will happen as long as the elasticity of substitution is larger than one. Of course, what matters is not the elasticity of substitution among inputs but among types. The latter depends on the form of the production function and also on how different the initial endowments of the types are (in the extreme case where they are collinear the two types are perfect substitutes as production inputs). Thus, in analogy to the terminology used by Ostroy and Zame (1994) in another context, we could say that the example is characterized by a low level of "physical substitution."

(ii) Utility is not perfectly transferable across types. We already know that if it were then the Harsanyi value (= the Shapley TU-value) and the competitive solution would coincide. Thus, in line with the previous terminology, we could say that the example is also characterized by a low level of "economic substitution."

We may try to highlight the role of substitution in a different way. Namely, by analyzing what happens when the relative mass of the two types changes. Suppose that we begin at $x=(50,50)$. Then, by symmetry, the competitive and the Harsanyi solutions coincide and they both give the same payoff $\approx(0.154,0.154)$ to the two types.

Let us now move to $\bar{x}=(51,49)$, that is, type 2 becomes relatively scarce. As we have seen, the core-hence the competitive payoff-moves to $\bar{c} \approx$ $(0.148,0.160)$. Hence, as it is natural, individuals of type 2 gain from the relative scarcity of their input. But note that they gain a lot since, in spite of the aggregate reduction of input from 50 to 49 , the total utility of the type increases: $49 \times 0.160>50 \times 0.154$. This sort of strong reward to scarcity is typical of situations with underlying strong complementarity effects.

What is surprising, given its "fairness" motivation, is that the Harsanyi value for $\bar{x}=(51,49)$, which is $\approx(0.117,0.192)$, rewards scarcity even more pronouncedly! (All these effects, incidentally, are preserved in the smoothed out version of Appendix C.) From the technical point of view we may try to understand what happens by focusing on the optimal path $\mathbf{x}(\cdot)$ that realizes $\nabla P(\bar{x})$, where $P$ is the potential corresponding to $\bar{\lambda}=(1,1)$, the utilitarian weights at the competitive allocation. This path is represented in Fig. 6 where we also indicate the level curves of the potential function $P$. The $\bar{\lambda}$-egalitarian solution 


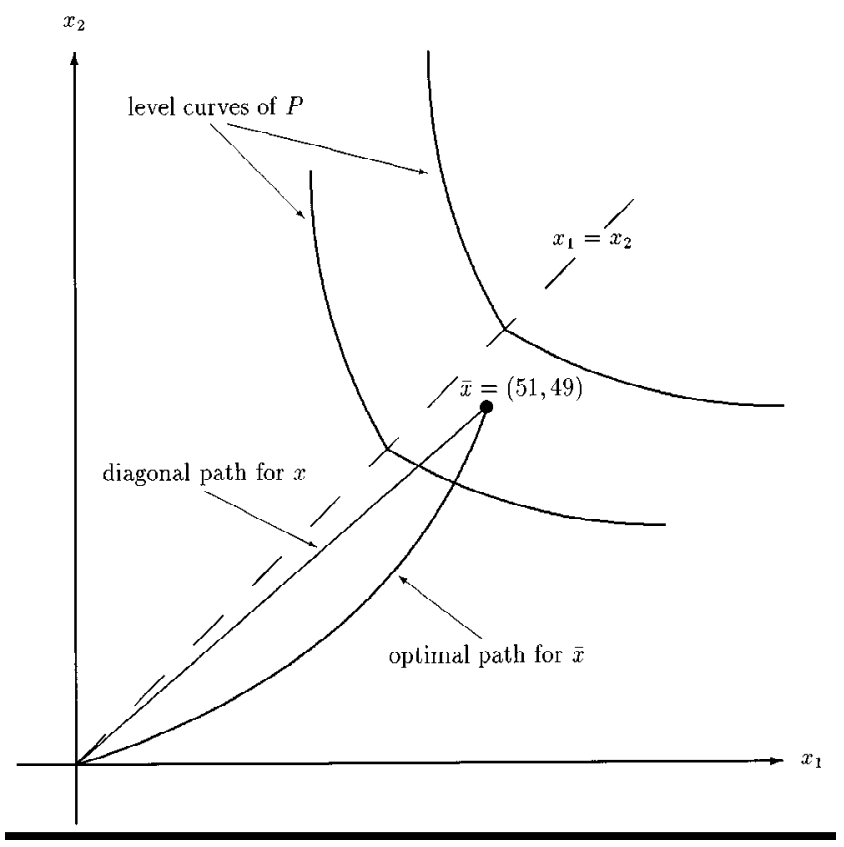

FIGURE 6

$\nabla P(\bar{x})$ is given by (see Theorem $\mathrm{E}(\mathrm{c})$ in $(\mathrm{HM}, \mathrm{I})$ ):

$$
\bar{x} * \nabla P(\bar{x})=\int_{0}^{1} \mathbf{x}(t) * \nabla_{x} \hat{v}(\mathbf{x}(t), \dot{\mathbf{x}}(t) / \mathbf{x}(t)) d t .
$$

Hence, $\nabla P(\bar{x})$ is an average of (properly computed) marginal productivities of the two types along the path. Because the path is not diagonal it follows that the marginal productivities change along the path and that therefore the "intra-marginal" units leave their mark in the egalitarian solution. In contrast, the competitive solution depends only on the marginal productivities at the "margin" $\bar{x}$, that is, on $\nabla_{x} \hat{v}(\bar{x}, \bar{\lambda})$ (recall Theorem VII.1 (C2)); equivalently, because of the homogeneity of degree one, on the marginal productivities along the diagonal path. We can see in Fig. 6 that along the optimal path the relative scarcity of type 2 is more pronounced than at the end point. Thus the average along the path-the $\bar{\lambda}$-egalitarian solution-magnifies the scarcity effect. At the Harsanyi value the weights $\lambda^{\prime}$ have adjusted in a countervailing direction, yet at the end an "excess scarcity" effect is still left.

We believe that, at the very least, the example presented casts some uncertainties on the value equivalence principle in the NTU context. But the matter 
deserves further investigation both within the theory of the Harsanyi value and beyond it. It would be of interest, for example, to examine carefully the extent of the validity of the equivalence principle for the consistent values of Maschler and Owen (1992) (see also Hart and Mas-Colell, 1996); note that these values admit a simple bargaining interpretation.

\section{APPENDIX A}

This appendix states the basic assumptions on the games studied in this paper. The reader is referred to our previous two papers, (HM,I and HM,II) for extensive discussions of them.

We first recall the notations; $a, b, p, q, x, y$ denote vectors in $\mathbb{R}^{n}$.

$$
\begin{aligned}
& \|x\|:=\sum_{i=1}^{n}\left|x_{i}\right| \\
& e=(1, \ldots, 1) \in \mathbb{R}^{n} \\
& x * y:=\left(x_{i} y_{i}\right)_{i=1, \ldots, n} \text { and } x / y:=\left(x_{i} / y_{i}\right)_{i=1, \ldots, n} \\
& \hat{V}(x):=\left\{b \in \mathbb{R}^{n}: b \leq x * a \text { for some } a \in V(x)\right\} \\
& \hat{V}_{0}(x):=\hat{V}(x) \cap \mathbb{R}_{+}^{n} \\
& v(x, p):=\sup \{p \cdot a: a \in V(x)\} \\
& \hat{v}(x, q):=\sup \{q \cdot b: b \in \hat{V}(x)\} \\
& \hat{v}_{0}(x, q):=\sup \left\{q \cdot b: b \in \hat{V}_{0}(x)\right\} \\
& D:=\left\{(x, p) \in \mathbb{R}_{++}^{n} \times \mathbb{R}_{+}^{n}: v(x, p)<\infty\right\} \\
& \hat{D}:=\left\{(x, q) \in \mathbb{R}_{++}^{n} \times \mathbb{R}_{+}^{n}: \hat{v}(x, q)<\infty\right\} \\
& \hat{D}^{+}:=\left\{(x, q) \in \hat{D}: \hat{v}(x, q)=q \cdot b \text { for some } b \in \hat{V}_{0}(x)\right\} .
\end{aligned}
$$

Now we state the basic assumptions. Note that the starred ones A.1*, A.2*, A. $3^{*}$, and A. $3 \mathrm{w}^{*}$, which are only needed for the finite approximation setup, concern all nonnegative profiles $x \in \mathbb{R}_{+}^{n}$, whereas all the rest apply only to strictly positive profiles $x \in \mathbb{R}_{++}^{n}$.

A.1. For every $x \in \mathbb{R}_{++}^{n}$, the set $V(x)$ is closed, convex, and comprehensive. Moreover, $V(x) \neq \emptyset$ and $V(x) \neq \mathbb{R}^{n}$.

A.1*. For every $x \in \mathbb{R}_{+}^{n}$, the set $V(x)$ is closed, convex, and comprehensive. Moreover, $V(x) \neq \emptyset$ and $V(x) \neq \mathbb{R}^{n}$. If $x_{i}=0$ and $a \in V(x)$ then $a^{\prime} \in V(x)$ whenever $a_{j}=a_{j}^{\prime}$ for all $j \neq i$.

A.2. There exists a $\theta>0$ such that $\theta e=(\theta, \theta, \ldots, \theta) \in V(x)$ for all $x \in \mathbb{R}_{++}^{n}$.

A.2* There exists a $\theta>0$ such that $\theta e=(\theta, \theta, \ldots, \theta) \in V(x)$ for all $x \in \mathbb{R}_{+}^{n}$. 
A.3. $\hat{V}_{0}(x)+\hat{V}_{0}\left(x^{\prime}\right) \subset \hat{V}_{0}\left(x+x^{\prime}\right)$ for all $x, x^{\prime} \in \mathbb{R}_{++}^{n}$.

A.3*. $\hat{V}_{0}(x)+\hat{V}_{0}\left(x^{\prime}\right) \subset \hat{V}_{0}\left(x+x^{\prime}\right)$ for all $x, x^{\prime} \in \mathbb{R}_{+}^{n}$.

A.3w. $\hat{V}_{0}(x)+\left\{x^{\prime} * \theta e\right\} \subset \hat{V}_{0}\left(x+x^{\prime}\right)$ for all $x, x^{\prime} \in \mathbb{R}_{++}^{n}$.

A. $3 \mathrm{w}^{*} . \quad \hat{V}_{0}(x)+\left\{x^{\prime} * \theta e\right\} \subset \hat{V}_{0}\left(x+x^{\prime}\right)$ for all $x, x^{\prime} \in \mathbb{R}_{+}^{n}$.

A.4. There is a compact set $C \subset\left\{q \in \mathbb{R}_{++}^{n}: \sum_{i} q_{i}=1\right\}$ such that $\hat{D} \subset$ $\mathbb{R}_{++}^{n} \times$ Cone $C$, where Cone $C:=\{\beta q: q \in C, \beta \geq 0\}$.

A.5. There exists a constant $K<\infty$ such that $\|b\| \leq K\|x\|$ for all $x \in \mathbb{R}_{++}^{n}$ and all $b \in \hat{V}_{0}(x)$.

A.6. The support function $\hat{v}_{0}(x, q)$ is uniformly Lipschitz on any bounded (not necessarily compact) subset of $\mathbb{R}_{++}^{n} \times$ Cone $C$; i.e., for every $\beta<\infty$ there is $K<\infty$ such that $\left|\hat{v}_{0}(x, q)-\hat{v}_{0}\left(x^{\prime}, q^{\prime}\right)\right| \leq K\left\|(x, q)-\left(x^{\prime}, q^{\prime}\right)\right\|$ for all $x, x^{\prime} \in \mathbb{R}_{++}^{n}$ with $\|x\|,\left\|x^{\prime}\right\| \leq \beta$ and all $q, q^{\prime} \in C$.

A.7. For every $x \in \mathbb{R}_{++}^{n}$ the support function $\hat{v}(x, \cdot)$ is strictly subadditive, i.e., $\hat{v}(x, q)+\hat{v}\left(x, q^{\prime}\right)>\hat{v}\left(x, q+q^{\prime}\right)$ whenever $q$ and $q^{\prime}$ are not collinear.

A.8. The gradient $\nabla_{x} \hat{v}_{0}(x, q)$ exists for all $(x, q) \in \mathbb{R}_{++}^{n} \times$ Cone $C$. Moreover,

(a) It is uniformly bounded on any bounded (not necessarily compact) subset of its domain $\mathbb{R}_{++}^{n} \times$ Cone $C$; i.e., for every $\beta<\infty$ there is $K<\infty$ such that $\left\|\nabla_{x} \hat{v}_{0}(x, q)\right\| \leq K$ for all $x \in \mathbb{R}_{++}^{n}$ with $\|x\| \leq \beta$ and all $q \in C$.

(b) It is uniformly Lipschitz on any bounded (not necessarily compact) subset of its domain $\mathbb{R}_{++}^{n} \times$ Cone $C$ which is bounded away from the origin of $\mathbb{R}_{++}^{n}$; i.e., for every $0<\rho \leq \beta<\infty$ there is $K<\infty$ such that $\left\|\nabla_{x} \hat{v}_{0}(x, q)-\nabla_{x} \hat{v}_{0}\left(x^{\prime}, q^{\prime}\right)\right\| \leq K\left\|(x, q)-\left(x^{\prime}, q^{\prime}\right)\right\|$ for all $x, x^{\prime} \in \mathbb{R}_{++}^{n}$ with $\rho \leq\|x\|,\left\|x^{\prime}\right\| \leq \beta$, and all $q, q^{\prime} \in C$.

A.9. The domain $\hat{D}^{+}$has nonempty interior and the function $\hat{v}(x, q)$ is $C^{2}$ on $\hat{D}^{+}$. Moreover, $\nabla_{q q} \hat{v}(x, q)$ has full possible rank $n-1$, and its minimal nonzero eigenvalue is positive and bounded aw ay from zero in a bounded (not necessarily compact) subset of $\hat{D}^{+}$that is bounded away from the origin of $\mathbb{R}_{++}^{n}$, i.e., on any set of the form $\hat{D}^{+} \cap\left(\left\{x \in \mathbb{R}_{++}^{n}: \rho \leq\|x\| \leq \beta\right\} \times C\right)$ for some $0<\rho \leq \beta<\infty$.

A.10. $V(t x)=V(x)$ for all $x \in \mathbb{R}_{++}^{n}$ and $t>0$.

All statements in the paper that refer to a continuum game $(\bar{x}, V)$ assume that $V$ satisfies ${ }^{13}$ A.1-A.9 and $\bar{x} \gg 0$. A continuum market game satisfies in addition

${ }^{13}$ For the results of Section IV one may replace A. $3 *$ by the weaker A.3w* (as is done in (HM, I) and (HM, II)). When we consider market games, we make use of the full superadditivity assumption A.3. 
A.10. In Section IV on the convergence of finite games, A.1-A.3 are (slightly) strengthened to A. $1^{*}-\mathrm{A} .3^{*}$.

\section{APPENDIX B}

This appendix contains the proof of Theorem IV.1. It uses arguments developed in (HM,I and HM,II).

Proof of Theorem IV.1. Let $a_{r} \in \partial V(\bar{x})$ be a Harsanyi value of $\left(N_{r}, V_{r}\right)$, corresponding to weights $\lambda_{r} \in C$. Assume without loss of generality that $a_{r} \rightarrow$ $a \in \partial V(\bar{x})$ and $\lambda_{r} \rightarrow \lambda \in C$ as $r \rightarrow \infty$. We will show that $a$ is a Harsanyi value of $(\bar{x}, V)$ corresponding to the weights $\lambda$. Since the utilitarian condition clearly holds, we need to prove that $a$ is $\lambda$-egalitarian.

Let $P_{r, \mu}$ denote the $\mu$-potential of the finite game $\left(N_{r}, V_{r}\right)$.Part (c) of the Main Theorem of $(\mathrm{HM}, \mathrm{II})$ states that, for every fixed $\mu \in C$, we have $\operatorname{dist}\left(D P_{r, \mu}(\bar{x}), \operatorname{Eg}_{\mu}(\bar{x})\right) \rightarrow 0$ as $r \rightarrow \infty$, where "dist" denotes distance and $\operatorname{Eg}_{\mu}(\bar{x}):=\left(\nabla^{c} P_{\mu}(\bar{x})+\mathbb{R}_{+}^{n}\right) \cap \partial V_{\mu}(\bar{x})$. Moreover, it can be easily checked that this convergence is uniform in $\mu$ (since all evaluations in (HM,II) are determined by the constants appearing in the various assumptions - and these are the same for all $\left.V_{\mu}\right)$. Therefore $\operatorname{dist}\left(\lambda_{r} * a_{r}, \operatorname{Eg}_{\lambda_{r}}(\bar{x})\right) \rightarrow 0$ as $r \rightarrow \infty$, and it remains to show that $\overline{\operatorname{Lim}} \operatorname{Eg}_{\lambda_{r}}(\bar{x})=\operatorname{Eg}_{\lambda}(\bar{x})$.

By the definition of $\operatorname{Eg}_{\mu}(\bar{x})$, it suffices to prove the convergence for the extreme points of $\nabla^{c} P_{\lambda_{r}}(\bar{x})$. Thus, let $x_{r} \rightarrow \bar{x}$ be such that $P_{\lambda_{r}}$ is differentiable at $x_{r}$ and $a_{r}:=\nabla P_{\lambda_{r}}\left(x_{r}\right) \rightarrow a$. Then, by Proposition VII.3.1 in (HM,I), $b_{r}:=x_{r} * a_{r}=\int_{0}^{1} \mathbf{x}_{r} * \nabla_{x} \hat{v}\left(\mathbf{x}_{r}, \lambda_{r} * \dot{\mathbf{x}}_{r} / \mathbf{x}_{r}\right)$, where $\mathbf{x}_{r}$ is (the) optimal path for $x_{r}$ and $\lambda_{r}$ (i.e., $P_{\lambda_{r}}\left(x_{r}\right)=\int v\left(\mathbf{x}_{r}, \lambda_{r} * \dot{\mathbf{x}}_{r}\right)$ ). Let $\mathbf{x}$ be a uniform limit of $\mathbf{x}_{r}$. As in the proof of Proposition VII.3.2 in(HM,I), we obtain that $\mathbf{x}$ is optimal for $\bar{x}$ and $\lambda$, that $\dot{\mathbf{x}}_{r} \rightarrow \dot{\mathbf{x}}$ in measure, and finally that $b_{r} \rightarrow \int_{0}^{1} \mathbf{x} * \nabla_{x} \hat{v}(\mathbf{x}, \lambda * \dot{\mathbf{x}} / \mathbf{x}) \in \bar{x} * \nabla^{c} P_{\lambda}(\bar{x})$ (see Proposition VII.4.4 in (HM,I)). But $b_{r} \rightarrow \bar{x} * a$, which completes the proof.

\section{APPENDIX C}

In this appendix we will show how to modify the example of Section VI in such a way that it will satisfy all the assumptions A.1-A.10, and still the unique core allocation is not a Harsanyi value.

The first, and a bit delicate, task consists in adjusting the example to a game for $V^{\varepsilon}$ similar to $V$ except that the two noncentral straight segments of the boundary of the sets $V(x)$ are replaced by smooth, strictly convex pieces.

To this effect, let $0<\varepsilon<1$. We define the game form $V^{\varepsilon}$ as follows: $\hat{V}^{\varepsilon}(x):=$ $g^{\varepsilon}(x) B^{\varepsilon}$ for every $x$, where $g^{\varepsilon}$ and $B^{\varepsilon}$ are $\varepsilon$-perturbations of $g$ and $B$, respectively. 
For $b_{1} \leq b_{2}$, let $h^{0}(b):=b_{1}+2 b_{2}, h^{1}(b):=\sqrt{\left(b_{1}\right)^{2}+\gamma\left(b_{2}\right)^{2}}$ (the constant $\gamma>0$ will be determined later) and then $h^{\varepsilon}(b):=(1-\varepsilon) h^{0}(b)+\varepsilon h^{1}(b)$. For $b_{1} \geq b_{2}$ make the symmetric construction relative to the diagonal $b_{1}=b_{2}$, namely $h^{\varepsilon}\left(b_{1}, b_{2}\right):=h^{\varepsilon}\left(b_{2}, b_{1}\right)$. We then define $g^{\varepsilon}(x):=h^{\varepsilon}(x * \nabla P(x)) \equiv$ $h^{\varepsilon}\left(x_{1} \nabla_{1} P(x), x_{2} \nabla_{2} P(x)\right)$ for all $x$, where $P$ is the potential (for $\lambda=(1,1)$ ) of the example of Section VI. Note that if $x_{1} \geq x_{2}$ then $x_{1} \nabla_{1} P(x) \leq x_{2} \nabla_{2} P(x)$, and thus we are in the case $b_{1} \leq b_{2}$ in the definition of $h^{\varepsilon}$. Moreover, the symmetry of $P$ and $h^{\varepsilon}$ along the corresponding diagonals implies the same for $g^{\varepsilon}$, i.e., $g^{\varepsilon}\left(x_{1}, x_{2}\right)=g^{\varepsilon}\left(x_{2}, x_{1}\right)$ for all $x$. Note that $g^{0}=g$ and that $g^{\varepsilon}$ and $h^{\varepsilon}$ are homogeneous of degree 1 . The constant $\gamma$ is chosen such that $\nabla_{1} g^{1}(1,1)=$ $\nabla_{2} g^{1}(1,1)$. Since the same equality holds for $g^{0}$, it will be satisfied also by all the $g^{\varepsilon}$. Therefore the two "pieces" of $g^{\varepsilon}$ are "glued" in a continuously differentiable way along the diagonal. Furthermore, the second derivatives also match there (use the Euler formula for $\nabla_{1} g^{1}$ and $\nabla_{2} g^{1}$ - which are homogeneous of degree 0 - at the point $(1,1))$. Thus $g^{\varepsilon}$ is actually a $C^{2}$ function. Also, it can be checked that the second derivatives of $g^{1}$ are bounded as $x$ approaches $(1,0)$ and $(0,1)$; hence $g^{\varepsilon}$ is concave for small enough $\varepsilon$ (recall that $g^{0}$ is concave). Finally, put

$$
B^{\varepsilon}:=\left\{b \in \mathbb{R}^{2}: h^{\varepsilon}\left(b_{1}, b_{2}\right) \equiv h^{\varepsilon}\left(b_{2}, b_{1}\right) \leq 1,3 b_{1}+3 b_{2} \leq 1.85\right\} .
$$

We can now check the following (compare with Section 6):

(1) The potential of $V^{\varepsilon}$ (for $\bar{\lambda}=(1,1)$ ) is the same function $P$ : for all $x$ we have $\nabla P(x) \in \partial V^{\varepsilon}(x)$ (Indeed, $g^{\varepsilon}$ was defined such that $h^{\varepsilon}(x * \nabla P(x))=$ $\left.g^{\varepsilon}(x)\right)$ and $P(x)=\int v(\mathbf{x}, \dot{\mathbf{x}})$ for some path $\mathbf{x}(t)$ ending at $x$. Therefore the egalitarian solution $\nabla P(\bar{x})$ is not on the boundary of $\partial V(\bar{x})$ with support $\bar{\lambda}=$ $(1,1)$, hence there is no Harsanyi value for $\bar{\lambda}=(1,1)$.

(2) The unique core allocation is proportional to $\nabla g^{\varepsilon}(\bar{x})$ (thus close to the vector $\bar{c})$; it lies on the boundary with support $\bar{\lambda}=(1,1)$.

We still have to deal with the straight piece of the boundary of $B^{\varepsilon}$ where $3 b_{1}+3 b_{2}=1.85$. But note that the points in this region (and in a neighborhood of it) do not arise at all in the considerations of (1) above. As for (2), only a neighborhood of the point $\bar{c}$ with supporting vector $\bar{\lambda}=(1,1)$ matters. We may therefore perturb the boundary of $B^{\varepsilon}$ around this central segment so as to make the boundary of $B^{\varepsilon}$ smooth and strictly convex. This will make all the assumptions A.1-A.10 hold. ${ }^{14}$

${ }^{14}$ Actually, for A.2 we will need to shift the origin: we replace $V^{\varepsilon}(x)$ by $V^{\varepsilon}(x)+\{\theta e\}$ for some fixed $\theta>0$. 


\section{REFERENCES}

Aumann, R. J. (1964). "Markets with a Continuum of Traders," Econometrica 32, 39-50.

Aumann, R. J. (1975). "Values of Markets with a Continuum of Traders," Econometrica 43, 611-646.

Aumann, R. J., and Shapley, L. S. (1974). Values of Non-Atomic Games. Princeton, NJ: Princeton Univ. Press.

Champsaur, P. (1975). "Cooperation vs. Competition,” J. Econ. Theory 11, 394-417.

Cheng, H. (1996). "Values of Perfectly Competitive Economies," in Handbook of Game Theory with Economic Applications, Vol. III (R. J. Aumann and S. Hart, Eds.), Elsevier, forthcoming.

Clarke, F. (1983). Optimization and Non-Smooth Analysis. New York: Wiley/Interscience.

Harsanyi, J. C. (1963). “A Simplified Bargaining Model for the $n$-Persons Cooperative Game," Int. Econ. Rev. 4, 194-220.

Hart, S. (1977). "Values of Non-Differentiable Markets with a Continuum of Traders," J. Math. Econ. 4, 103-116.

Hart, S., and Mas-Colell, A. (1989). "Potential, Value, and Consistency," Econometrica 57, 589-614. Hart, S., and Mas-Colell, A. (1996). "Bargaining and Value," Econometrica 64.

Hart. S., and Mas-Colell, A. (1995a). "Egalitarian Solutions of Large Games. I. A Continuum of Players," Math. Oper. Res. 20, 959-1002. Referred to as (HM,I).

Hart. S., and Mas-Colell, A. (1995b). "Egalitarian Solutions of Large Games. II. The Asymptotic Approach," Math. Oper. Res. 20, 1003-1022. Referred to as (HM,II).

Imai, H. (1983). “On Harsanyi’s Solution,” Int. J. Game Theory 12, 161-179.

Kalai, E., and Samet, D. (1985). "Monotonic Solutions to General Cooperative Games," Econometrica 53, 307-327.

Maschler, M., and Owen, G. (1992). "The Consistent Shapley Value for Games without Side Payments," in Rational Interaction (R. Selten, Ed.), pp. 5-12. New York/Berlin: Springer-Verlag.

Mas-Colell, A. (1977). “Competitive and Value Allocations of Large Exchange Economies,” J. Econ. Theory 14, 419-438.

Myerson, R. (1980). “Conference Structures and Fair Allocation Rules,” Int. J. Game Theory 9, 169182.

Nash, J. (1950). “The Bargaining Problem,” Econometrica 18, 155-162.

Ostroy, J., and Zame, W. (1994). "Non-Atomic Economies and the Boundaries of Perfect Competition," Econometrica 62, 593-633.

Roth, A. (1980). "Values for Games without Side Payments: Some Difficulties with Current Concepts," Econometrica 48, 457-465.

Shafer, W. (1980). "On the Existence and Interpretation of Value Allocations," Econometrica 48, 467-477.

Shapley, L. S. (1953). "A Value for $n$-Person Games," in Contributions to the Theory of Games II, Annals of Mathematics Studies, Vol. 28 (H. Kuhn and A. Tucker, Eds.), pp. 307-317. Princeton, NJ: Princeton University Press.

Shapley, L. S. (1969). "Utility Comparison and the Theory of Games," in La Decision, pp. 251-263, Paris: Editions du CNRS.

Shapley, L. S., and Shubik, M. (1969). "Pure Competition, Coalitional Power, and Fair Division," Int. Econ. Rev. 69, 337-362. 